\section{Unusual Site of Deep Vein Thrombosis After mRNA Coronavirus Disease-2019 (COVID-19) Vaccination}

Tomoya Hasegawa, MD; Yoshikazu Yazaki, PhD

$\mathbf{T}$ he coronavirus disease-2019 (COVID-19) pandemic continues to pose a threat globally, and safe, effective vaccination is essential to resolve this public health issue. Venous thromboembolism (VTE) complications reportedly occur in unusual sites after ChAdOx2 nCoV-19 (AstraZeneca) vaccination, ${ }^{1}$ but few reports on the development of VTE after mRNA COVID-19 vaccination (Pfizer-BioNTech) have been published. ${ }^{2}$

A 72-year-old Japanese woman presented with persistent left arm claudication after receiving the mRNA COVID-19 vaccine. Both doses were injected in the left arm. She developed acute left neck and upper extremity swelling, accompanied by pain at rest, 28 days after the second dose was administered. She had an unremarkable medical history. There was no history of trauma, surgery or thrombotic events. She received no long-term medications. Blood tests revealed a normal platelet count, coagulation profile, and C-reactive protein level. Enhanced computed tomography showed thrombotic occlusion of the left subclavian vein that progressed into the left jugular vein (Figure A-C). Thrombophilia screening with proteins $\mathrm{C}$ and $\mathrm{S}$, antiphospholipid antibodies, and antibody to platelet-factor 4 were negative. ${ }^{18} \mathrm{~F}$-fluoro-deoxy-glucose positron emission tomography/ computed tomography was performed because a malignancy was suspected. The left supraclavicular region exhibited a mildly increased uptake suggestive of inflammation (Figure D). Low-dose apixaban therapy was initiated, and her symptoms gradually resolved.
This case highlights the rare occurrence of VTE in an uncommon site, related to mRNA COVID-19 vaccination. No other possible etiologies were identified. Although VTE reportedly occurs within 4-16 days after COVID-19 vaccination, ${ }^{3}$ it occurred later in this case. Therefore, a longer follow-up period after mRNA COVID-19 vaccination is needed.

\section{Disclosures}

The authors declare no conflicts of interest.

\section{IRB Information}

This study was approved by the Saku Central Hospital Group Research Ethics Committee (reference no. R202110-01).

\section{Reference}

1. Schultz NH, Sørvoll IH, Michelsen AE, Munthe LA, LundJohansen F, Ahlen MT, et al. Thrombosis and thrombocytopenia after ChAdOx1 nCoV-19 vaccination. $N$ Engl J Med 2021; 384: 2124-2130.

2. Carli G, Nichele I, Ruggeri M, Barra S, Tosetto A. Deep vein thrombosis (DVT) occurring shortly after the second dose of mRNA SARS-CoV-2 vaccine. Intern Emerg Med 2021; 16: 803-804.

3. Oldenburg J, Klamroth R, Langer F, Albisetti M, Auer CV, Ay C, et al. Diagnosis and management of vaccine-related thrombosis following AstraZeneca COVID-19 vaccination: Guidance statement from the GTH. Hamostaseologie 2021; 41: 184-189.

Received October 18, 2021; revised manuscript received October 25 , 2021; accepted October 26, 2021; J-STAGE Advance Publication released online November 27, 2021 Time for primary review: 3 days

Division of Cardiology, Saku Central Hospital Advanced Care Center, Saku, Japan

Mailing address: Tomoya Hasegawa, MD, Division of Cardiology, Saku Central Hospital Advanced Care Center, 3400-28 Nakagomi, Saku385-0051, Japan. E-mail: tomoya.hasegawa0825@gmail.com All rights are reserved to the Japanese Circulation Society.

For permissions, please e-mail: cj@j-circ.or.jp ISSN-1346-9843
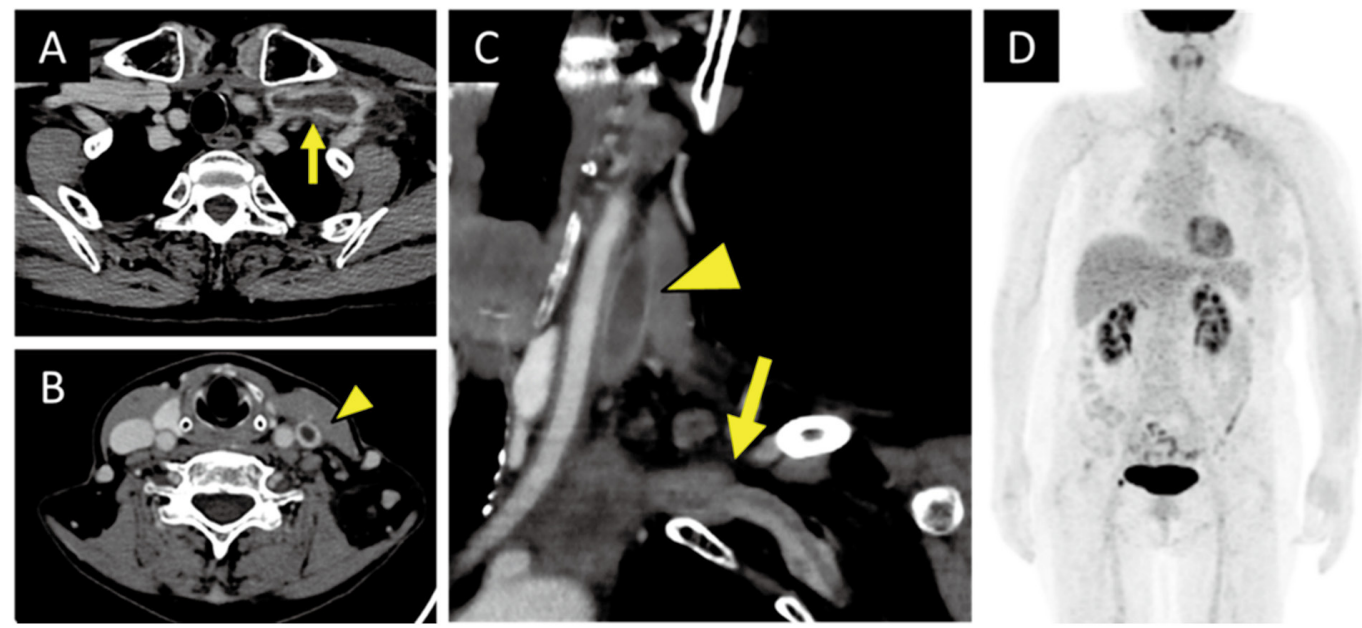

Figure. (A-C) Enhanced computed tomography shows occlusion of the left subclavian vein (arrow) and jugular vein (arrowhead). (D) ${ }^{18} \mathrm{~F}$-fluoro-deoxy-glucose positron emission tomography/computed tomography image does not show intense uptake of ${ }^{18}$ F-fluoro-deoxy-glucose. 